\title{
Método modificado de muestreo por conglomerados para la evaluación rápida de necesidades después de un desastre ${ }^{1}$
}

\author{
J. Malilay, ${ }^{2}$ W. D. Flanders ${ }^{3}$ y D. Brogan ${ }^{3}$
}

RESUMEN El método de muestreo por conglomerados puede utilizarse para llevar a cabo la evaluación rápida de las necesidades de salud y de otro tipo en comunidades afectadas por desastres naturales. El método, que se basa en el modelo usado por el Programa Ampliado de Inmunización de la OMS para estimar la cobertura con vacunación, ha sido modificado para que proporcione 1) estimaciones de la población que queda en una zona determinada y 2) estimaciones del número de personas con necesidades específicas en la zona que ha sido afectada por un desastre. Este enfoque difiere del que se ha usado anteriormente a raíz de otros desastres en que las evaluaciones rápidas de las necesidades solo han consistido en estimar la proporción de la población con necesidades específicas. Aquí se propone un diseño de encuesta modificado que se basa en el $u$ so de $n \times k$ para calcular la población restante, la gravedad del daño, la proporción y el número de personas con necesidades específicas, el número de domicilios dañados o destruidos y los cambios que sufren estas estimaciones en determinado período como parte de la encuesta.

La evaluación rápida de necesidades, que también se conoce por evaluación rápida sanitaria o epidemiológica, se refiere a una colección de técnicas epidemiológicas, estadísticas y antropológicas diseñadas para proporcionar a los formuladores de políticas, de manera rápida y barata, información poblacional acertada en un formato sencillo $(1,2)$. Se ha visto que el método es útil e importante para determinar las necesidades inmediatas

\footnotetext{
1 Se publicó en inglés en el Bulletin of the World Health Organization, Vol. 74, No. 4, 1996, con el título "A modified cluster-sampling method for post-disaster rapid assessment of needs". (C) Organización Mundial de la Salud, 1996.

2 Centros para el Control y la Prevención de Enfermedades, Atlanta, Georgia, EUA. Dirección postal: Disaster Assessment and Epidemiology, CDC, 4770 Buford Highway, NE(Mailstop F-46), Atlanta, GA 30341-3724.

3 Universidad de Emory, Atlanta, Georgia, EUA.
}

en el campo de la comunicación después de urgencias, tales como los desastres naturales $(3,4)$. Anteriormente se ha aplicado para varios fines, entre ellos la evaluación de estado nutricional, mortalidad, morbilidad y acceso a los campamentos y servicios salvavidas después de urgencias que afectan a grupos de refugiados y a la población $(5,6)$ y después de desastres naturales (7-9).

El método de muestreo por conglomerados puede usarse para evaluar rápidamente las necesidades de las comunidades afectadas después de un desastre natural. El método de muestreo por conglomerados se aplicó, por ejemplo, en tres encuestas para la evaluación de necesidades después de la llegada del huracán Andrew a la Florida en 1992 (9). Para estas encuestas se usó de modelo el método del Pro- grama Ampliado de Inmunización (PAI) de la OMS, que se diseñó para estimar la cobertura por inmunización. Las encuestas proporcionaron información demográfica y de otro tipo sobre la composición familiar, las heridas y enfermedades sufridas en el momento del trauma y en el período posterior, la disponibilidad de medicamentos, las fuentes de agua y electricidad, y el estado de los medios de comunicación y transporte, e indicaron las áreas de mayor prioridad para que los encargados de las medidas de auxilio pudieran concentrarse en dar una respuesta apropiada y efectiva. De acuerdo con la OMS, este diseño por conglomerados es fácil de poner en práctica en el terreno, requiere pocos recursos y arroja estimaciones bastante válidas y exactas para análisis y difusión a un ritmo relativamente 
acelerado. Con este diseño, los intervalos de confianza de $95 \%$ nunca pasan de $20 \%$ (estimación puntual, más o menos $10 \%$ ) si se estudian 30 conglomerados con siete individuos cada uno, siempre que el efecto del diseño no sea mayor de 2,0 (10). Desde sus primeras aplicaciones en los años ochenta, el método ha sido modificado para que rinda datos más exactos que permitan evaluar los programas del PAI (11).

La experiencia que se ha adquirido en situaciones de desastre ha demostrado, sin embargo, que tales evaluaciones deben proporcionar estimaciones del número y no solo del porcentaje de personas que necesitan cierto tipo de ayuda en el lugar del desastre. Por añadidura, en ocasiones es necesario repetir las encuestas debido al cambio de necesidades a medida el desatre avanza.

En este trabajo se describe una modificación del método de muestreo por conglomerados que se puede utilizar para proporcionar la siguiente información:

- estimaciones de la población que queda en la zona, que puede ser distinta de la que había antes del desastre y que puede cambiar a lo largo del tiempo, a medida que la comunidad afectada se desplaza hacia lugares con disponibilidad de servicios públicos;

- estimaciones del número de personas en la zona que tienen necesidades particulares después del desastre.

Este enfoque difiere de los que se han usado previamente para hacer encuestas después de desastres, en que habitualmente se ha calculado solo la proporción con necesidades específicas. También se examinan otros puntos relacionados con la evaluación rápida de las necesidades.

\section{MATERIALES Y MÉTODOS}

Aquí se propone un diseño de encuesta modificado a base del cálculo de $n \times k$ en que se estiman la población restante, la gravedad de los daños, la proporción y el número de personas con necesidades específicas, el número de unidades domiciliarias (UD) dañadas o destruidas y los cambios que sufren las estimaciones a lo largo del tiempo como parte de la encuesta.

\section{Procedimiento}

- Se divide el lugar del desatre en varios bloques o conglomerados generales completamente separados el uno del otro. Cada bloque debe ser lo suficientemente pequeño para que el número total de domicilios en cada bloque seleccionado se pueda contar. La división puede hacerse siguiendo el plano cuadriculado de las calles, si está disponible, o siguiendo las fronteras geográficas naturales, tales como los ríos y colinas identificados en los mapas de la topografía local. Cada bloque o conglomerado debe tener límites bien definidos para que el personal pueda identificarlo sobre el terreno si se decide incluirlo en la muestra.

- Se hace una estimación preliminar del número de UD en cada bloque utilizando la información proporcionada por el censo, mapas aéreos, datos proporcionados por las autoridades locales u otros recursos disponibles. Se denomina con el símbolo $H_{i}^{\prime}$ este estimado preliminar del número de UD en el conglomerado $i$, y con el símbolo $H^{\prime}$ el número estimado de domicilios en la zona, de tal manera que

$$
H^{\prime}=\sum_{i=1}^{N} H_{i}^{\prime} n
$$

y $N$ es el número total de bloques o conglomerados en la zona del desastre, normalmente denominados unidades de muestreo primarias (UMP).

- Se escoge una muestra de $n$ bloques cuya probabilidad esté en proporción con el número estimado de domicilios. En el método del PAI se usan $n=30$ conglomerados. En muchos casos, se aplica un muestreo probabilístico sistemático, en proporción con el tamaño, cuando se tiene un marco muestral en que las UMP o los bloques están ordenados por proximidad geográfica.

- Dentro de cada conglomerado de la muestra, se cuentan (y se hace una lista, si es posible) todas las UD; el número total se denota con la expresión $H_{i}$. También se cuenta e indica el número de UD destruidas, que se denomina con la expresión $D_{i}$.

- Si se da por sentado que todos los domicilios en un bloque determinado han sido incluidos en la lista, se escoge una muestra equiprobabilística, sin reemplazo, de $k_{i} \mathrm{UD}$. A menudo se usa un muestreo aleatorio sistemático cuando los domicilios están ordenados por proximidad geográfica. Con el método del PAI se escoge el número de UD que sea necesario para identificar a siete individuos dentro de un conglomerado muestral, pero aquí se recomienda elegir un número fijo, $k_{i}$, de domicilios en cada conglomerado de la muestra (11). Sin embargo, cuando un conglomerado que ha sido escogido es demasiado grande para que se enumeren todas las UD que contiene, se hace un recuento aproximado de ellas. Posteriormente se aplica el procedimiento de división en sectores para escoger un sector dentro de cada conglomerado, como describen Brogan et al. (11).

- Se cuenta el número de personas que vive en cada una de las UD escogidas; se denomina con el símbolo $C_{i j}$ el número de personas que viven en la UD $j$ de la UMP o bloque $i$. Se administra el cuestionario a un miembro de cada UD seleccionada que sea capaz de contestar las preguntas sobre la composición del hogar y las necesidades de sus integrantes. Esta persona no tiene que elegirse al azar. De forma similar, se denota con el símbolo $R_{i j}$ el número total de personas en la UD $j$ del bloque $i$ que tienen una necesidad específica.

- Si no hay nadie en casa, se identifica a un vecino para obtener información sobre la UD seleccionada o se regresa más tarde cuando sea más probable que alguien se encuentre en casa. Se debe procurar conseguir información sobre los habitantes de 
cada UD seleccionada al azar. No recomendamos que se reemplacen con nuevas UD de la muestra las UD muestrales cuyos habitantes no se encuentran en casa. Las UD de la muestra que estén vacías (desocupadas) se anotan en la lista de la siguiente manera: $C_{i j}=R_{i j}=0$.

El cálculo de la población. La población total después del desastre se define como sigue:

$$
\mathrm{C}=\sum_{i=1}^{N} \sum_{j=1}^{H_{i}} C_{i j}
$$

La estimación puntual de C es:

$$
\hat{\mathrm{C}}=\sum_{i=1}^{n} \sum_{j=1}^{k_{i}} w_{i} C_{i j}
$$

donde $w_{i}=(1 / n) \times\left(H^{\prime} / H_{i}^{\prime}\right) \times\left(H_{i} / K_{i}\right)$ y $k_{i}$ representa el número de UD del bloque $i$ que en realidad se han incluido en la muestra.

La varianza estimada aproximada (12) de esta población total calculada está dada por:

$\operatorname{Var} r(\hat{C}) \cong \frac{n}{n-1} \sum_{i=1}^{n}\left(w_{i} \sum_{j=1}^{k_{i}} C_{i j}-\hat{C} / n\right)^{2}$

El cálculo de las necesidades. Asimismo, el número total de habitantes de la zona que tienen una

$$
R=\sum_{i=1}^{N} \sum_{j=1}^{H_{i}} R_{i j}
$$

necesidad particular, denominado por $R$, se define así:

$$
\hat{R}=\sum_{i=1}^{n} \sum_{j=1}^{k_{i}} W_{i} R_{i j}
$$

La estimación puntual de $\hat{R}$ es:

La varianza estimada de $R$ está dada por la ecuación (4), si se sustituye Rij por Cij y $\mathrm{R}$ por $\mathrm{C}$.

El cálculo del número de unidades domiciliarias destruidas. El número total de UD destruidas se define de la siguiente forma:

$$
D=\sum_{i=1}^{N} D_{i}
$$

La estimación puntual de $D$ es:

$$
\hat{D}=\sum_{i=1}^{n} W_{i} D_{i}
$$

donde $W_{i}=(1 / n) \times\left(H^{\prime} / H_{i}^{\prime}\right)$.

La varianza estimada aproximada de este número total de UD destruidas está dada por:

$$
\operatorname{Var}(\hat{D}) \cong \frac{n}{n-1} \sum_{i=1}^{n}\left(W_{i} D_{i}-\hat{D} / n\right)^{2}
$$

\section{Suposiciones}

Las suposiciones que se hicieron para derivar las fórmulas de la estimación puntual y del cálculo de la varianza se enumeran a continuación.

- Los domicilios incluidos en el marco muestral no se repiten y abarcan la zona geográfica que interesa, es decir, la zona del desastre.

- El muestreo de los domicilios (o bloques) en la primera etapa se ha hecho con reemplazo o, en los casos en que no se ha hecho un reemplazo, $n$ es un porcentaje pequeño de $N$, de tal modo que no es importante usar el factor de corrección para poblaciones finitas en la estimación de la varianza.

- El muestreo de la primera etapa no es equiprobabilístico, es decir, que la probabilidad está en proporción con el tamaño. No obstante, las fórmulas siguen siendo útiles si se hace equiprobabilístico el muestreo de los bloques o conglomerados en la primera etapa.

- En la segunda etapa el muestreo de las UD $k_{i}$ dentro de la UMP $i$ incluida en la muestra es equiprobabilístico.

- Se obtiene información sobre todos los miembros de la UD incluida en la muestra.

- El número de UD incluidas en la muestra es lo suficientemente grande para que sea válida la varianza aproximada [ecuación (4)]. Se recomienda obtener una muestra de 30 domicilios (13).
Otras cuestiones relacionadas con el trabajo sobre el terreno

Las encuestas estratificadas. Las encuestas se pueden estratificar según cualquier variable pertinente, como sería la gravedad de los daños. Debido al hecho de que los desastres pueden afectar de forma desigual a áreas distintas dentro de la zona afectada, se pueden hacer encuestas separadas en diferentes zonas, como en aquellas donde los daños fueron pocos, moderados o grandes. Estas necesidades heterogéneas pueden deberse a variaciones del diseño y de la construcción de las viviendas, a la existencia de sistemas de alarma o a la ubicación geográfica. Las encuestas separadas o estratificadas, como las que se hacen para calcular la magnitud del daño, pueden proporcionar una idea general de las necesidades existentes en las comunidades afectadas.

Si en una encuesta estratificada se desea estimar los parámetros poblacionales de cada estrato, se necesita por estrato una muestra de 30 UMP. Se pueden combinar los datos de algunos estratos (o todos), haciendo las ponderaciones adecuadas, para calcular los parámetros poblacionales de una parte de la zona geográfica o de la zona en su totalidad.

Aunque no se desee estimar los parámetros de población de cada estrato, puede que sí se quiera estratificar las UMP y usar un muestreo aleatorio estratificado para asegurar que cada estrato esté representado en la muestra. En este caso, el tamaño muestral no tiene que ser de 30 UMP por estrato, sino de 30 UMP o más en total. Si se hace la estratificación, las fórmulas dejan de ser válidas. En el anexo 1 se presentan las modificaciones de las fórmulas que deben hacerse cuando la encuesta es estratificada.

La repetición de encuestas a lo largo del tiempo. Debido a que las necesidades después de un desastre varían a medida que pasa el tiempo, las encuestas deben repetirse a fin de detectar cambios de las necesidades. Por ejemplo, inmediatamente después del 
siniestro las necesidades gravitan en torno a actividades de búsqueda y rescate, primeros auxilios y abastecimiento de agua y alimentos. No obstante, a los 3 días las actividades de respuesta pueden dirigirse a establecer viviendas improvisadas y sistemas de vigilancia epidemiológica para la población reunida en campamentos. Una semana después del desastre, las prioridades de la fase de auxilio y rescate pueden desplazarse hacia la restauración de los medios de comunicación y transporte y de otros sistemas salvavidas (14).

Lo ideal es hacer una encuesta inmediatamente después del desastre y repetirla a los pocos días o quizá semanalmente a lo largo de toda la recuperación, por un período de alrededor de un mes. Para las encuestas de seguimiento se podría hacer un nuevo muestreo de las UMP y, si las poblaciones cambian después del desastre, se podrían cambiar también las probabilidades de selección. Otra opción sería la de volver a los mismos domicilios para hacer cada encuesta de seguimiento. Esta opción proporcionaría información directa sobre los subsiguientes cambios de necesidades pero complicaría la interpretación y el análisis si, por ejemplo, algunos de los domicilios que estaban ocupados durante la primera encuesta ya no lo estuvieran durante la segunda. La interpretación también sería difícil si las UMP que no estaban habitadas durante la primera encuesta tampoco lo estuvieran durante la segunda.

Muestreo sin reemplazo y con fracción muestral grande durante la primera etapa. Si se lleva a cabo un muestreo sin reemplazo y es grande la fracción de los conglomerados que se incluyeron en la muestra elegida en la primera etapa (e.g., $n / N>0,05$ ), puede ser que el factor de corrección para poblaciones finitas no sea insignificante. En ese caso, la ecuación (4) tiende a sobreestimar la varianza. En los casos sencillos en que el muestreo de los conglomerados es equiprobabilístico en la primera etapa, la varianza estimada después de usar el factor de corrección para poblaciones finitas se presenta en el anexo 2. (Las fórmulas usadas para la estimación puntual son las mismas que se indican en el texto.)

La estimación de la varianza, que incluye el factor de corrección para poblaciones finitas cuando el muestreo en la primera etapa no es equiprobabilístico, resulta complicada (12). No obstante, el uso de esta ecuación compleja se puede evitar usando un muestreo con reemplazo o un gran número de conglomerados y tomando una muestra pequeña de ellos (e.g., menos de 5 a $10 \%$ ).

\section{DISCUSIÓN}

La modificación que proponemos es distinta del método de muestreo del PAI y de los métodos de muestreo por conglomerados usados previamente en situaciones de desastre. El método del PAI exige la selección aleatoria de una UD dentro de un conglomerado o punto inicial. La selección de individuos comienza en el punto inicial y continúa hasta que se consigue a siete individuos - cuyas edades interesan en función de su estado de inmunización- en las UD que siguen por orden de proximidad. En el último domicilio se suman todas las personas que están en el grupo de edad deseado, de manera que un conglomerado puede contener más del número mínimo de siete individuos (10). Cuando se aplicó el diseño por conglomerados después del desastre causado por el huracán Andrew en los Estados Unidos de América, los entrevistadores llegaron a un punto cerca del centro de cada uno de los 30 conglomerados, se encaminaron en una dirección elegida al azar (por lanzamiento de moneda) hasta llegar a la UD habitada más cercana y entrevistaron a un habitante adulto de la UD. Después siguieron caminando a la próxima UD hasta que completaron siete entrevistas con habitantes de las UD ocupadas.

Las UD deshabitadas no fueron visitadas. En el caso de viviendas compartidas por varias familias, solamente se entrevistó a las personas de la primera UD habitada. Si un conglomerado no se estaba usando para fines residenciales o había quedado destruido, los entrevistadores continuaban hasta el conglomerado más cercano que estuviera situado en una dirección elegida al azar (9). Además de la proporción y el número de personas con necesidades específicas, el método aquí propuesto también sirve para calcular la población restante y el número de UD dañadas o destruidas.

Según demuestran estos resultados, la simple modificación del método de muestreo por conglomerados utilizado por el PAI puede proporcionar información sobre el tamaño de la población que queda después de un desastre y la magnitud de sus necesidades. Puede que haga falta más de una encuesta o que se necesite una encuesta estratificada si es grande la zona afectada o si los daños son heterogéneos. Es posible que se necesiten encuestas de seguimiento para evaluar los cambios de necesidades que se producen en determinado período.

Hasta la fecha, el diseño por conglomerados se ha usado después de desastres que han dejado daños muy extensos, como los ciclones tropicales $(7,9)$. Después de otros tipos de desastres, como los terremotos, ciertas partes de la zona del siniestro pueden quedar más afectadas que otras, como en aquellas zonas donde se construyeron edificios antiguos antes de que se impusieran reglamentos estrictos sobre las estructuras resistentes a los terremotos. Una encuesta completa (es decir, con una muestra de 100\%) de todas las zonas afectadas puede resultar más apropiada si el daño se circunscribe a determinados lugares solamente o a vecindarios ubicados dentro de la zona del desatre.

Dado que la información debe ser oportuna y estar a disposición de las autoridades en poco tiempo, los aspectos logísticos de una evaluación rápida de las necesidades merecen cuidadosa atención. Debe formularse un cuestionario breve a fin de que se pueda llenar con rapidez; los organizadores de la encuesta deben cerciorarse de que haya suficientes personas en los equipos encargados de calcular las necesidades para que se lleven a cabo por lo 
menos 210 entrevistas (30 bloques $\times 7$ domicilios). El número $k^{i}$ de domicilios que ha de seleccionarse para la muestra puede ser mayor de siete para compensar por los domicilios deshabitados. Si el objetivo principal es estimar C y $R$, los domicilios deshabitados sí proveen información, ya que 0 personas viven en ellos y las necesidades correspondientes son de 0 . Por consiguiente, no sería necesario aumentar el número $k_{i}$ de domicilios. Sin embargo, si el objetivo principal es estimar la proporción de personas que tienen necesidades, resulta importante tener siete domicilios habitados en cada bloque. Por ejemplo, si los investigadores hacen una estimación preliminar según la cual $75 \%$ de los domicilios estarán deshabitados, el valor de $k_{i}$ es de 7/0,75. De esta forma, un número aproximado de siete domicilios habitados debe proveer información sobre las necesidades en cada conglomerado. Es preciso incluir en los cálculos períodos de tiempo razonables para el proceso de recolectar datos: es decir, calcular períodos más breves en zonas urbanas donde los domicilios suelen estar cercanos los unos a los otros, y más largos en sitios rurales remotos donde los domicilios están más separados.

Por último, otros datos pueden incluirse en la evaluación. Para determinar la gravedad del daño, una evaluación de necesidades puede proveer estimaciones de 1) el número o porcentaje de UD destruidas y 2) el número o porcentaje de UD habitables. Una evaluación rápida también puede proporcionar un cálculo de la morbilidad y mortalidad relacionadas con el desastre. Esta información sería especialmente útil en zonas donde no hay registros estadísticos o donde estos registros son de mala calidad. La evaluación rápida de la situación de salud aguda de subgrupos en alto riesgo, como serían las personas afectadas por las enfermedades respiratorias o diarreicas que suelen ocurrir después de desastres geológicos o hidrometeorológicos, puede incluirse a fin de determinar si hay alguna desviación de los niveles endémicos. Una evaluación rápida de necesidades puede también incluir la toma de muestras ambientales para detectar cualquier exposición a agentes tóxicos y sus consecuencias para la salud. Sirven de ejemplo la toxicidad pulmonar producida por las partículas de ceniza que quedan suspendidas en el aire después de una erupción volcánica y la enfermedad gastrointestinal causada por aguas subterráneas contaminadas con agentes biológicos o químicos después de inundaciones.

Las evaluaciones iniciales de las zonas afectadas por un desastre pueden basarse en fotografías aéreas o informes verbales. Estas evaluaciones preliminares pueden revelar el área

\section{REFERENCIAS}

1. Anker M. Epidemiological and statistical methods for rapid health assessment: introduction. World Health Stat Q 1991;44:94-97.

2. Smith GS. Development of rapid epidemiologic assessment methods to evaluate health status and delivery of health services. Int $J$ Epidemiol 1989;18:S2-S15.

3. Guha-Sapir D. Rapid assessment of health needs in mass emergencies: review of current concepts and methods. World Health Stat $Q$ 1991;44:171-181.

4. Lillibridge SR, Noji EK, Burkie FM. Disaster assessment: the emergency health evaluation of a population affected by a disaster. Ann Energ Med 1993;22:1715-1720.

5. de Ville de Goyet C, Seaman J, Geijer U. The management of nutritional emergencies in large populations. Geneva: World Health Organization; 1978.
6. Toole MJ. The rapid assessment of health problems in refugee and displaced populations. Med Glob Surv 1994;1:200-207.

7 Sommer A, Mosley WH. East Bengal cyclone of November, 1970: epidemiological approach to disaster assessment. Lancet 1972;1: 1029036.

8. Centers for Disease Control and Prevention. Rapid needs assessment following Hurricane Andrew - Florida and Louisiana, 1992. MMWR 1992;41:685-688.

9. Hlady WG, et al. Use of a modified cluster sampling method to perform rapid needs assessment after Hurricane Andrew. Ann Emerg Med 1994;23: 719-725.

10. Henderson RH, Sundaresan T. Cluster sampling to assess immunization coverage: a review of experience with a simplified sampling method. Bull World Health Organ 1982;60:253-260. que se debe encuestar y también la necesidad de efectuar más de una encuesta si la zona es grande y el daño es muy extenso. Otra opción es dejar que la evaluación de las necesidades sea tarea de equipos encargados de evaluar los daños y reestructurar la selección de los conglomerados para la próxima encuesta (posiblemente independiente), cuando se sepa la extensión del daño. Por último, la encuesta solo puede abarcar zonas accesibles, ya que algunos de los conglomerados seleccionados en la primera etapa no serán accesibles. Por consiguiente, las estimaciones efectuadas en estas áreas no serían representativas de la población de toda la zona que se escogió inicialmente para hacer la encuesta.

En resumen, esta modificación del método de muestreo por conglomerados usado por el PAI puede aplicarse para obtener estimaciones razonablemente acertadas y válidas de las poblaciones que quedan después de los desastres y de la magnitud de sus necesidades a lo largo de un período determinado.

Agradecimiento. El trabajo de W. D. Flanders fue subvencionado por la Asociación de Escuelas de Salud Pública y por los Centros para el Control y la Prevención de Enfermedades de los Estados Unidos de América.
11. Brogan D, et al. Increasing the accuracy of the expanded programme on immunizations cluster survey design. Ann Epidemiol 1994;4: 302-311.

12. Shah BV, et al. Statistical methods and mathematical algorithms used in SUDAAN. Research Triangle Park, NC, Research Triangle Institute, 1993.

13. Cochran WG. Sampling techniques, 3a ed. New York: Wiley; 1978.

14. Pan American Health Organization. Assessing needs in the health sector after floods and hurricanes. Washington, DC: PAHO; 1987. (Documento técnico 11). 


\section{ANEXO 1}

\section{Muestra estratificada por conglomerados}

Se definen los conglomerados como se hizo anteriormente. Se forman $G$ grupos (o estratos) de conglomerados con $N_{\mathrm{g}}$ conglomerados en cada grupo $g, g=1,2, \ldots$, G. Estos grupos se pueden formar después de una evaluación preliminar de los daños que ha sufrido la zona, de tal manera que los daños y necesidades dentro de cada grupo sean relativamente homogéneos. Por ejemplo, un grupo puede haber sufrido daños leves a moderados, un segundo grupo daños moderados a intensos, y un tercer grupo los daños más graves. Los estratos también pueden definirse según parámetros geográficos, es decir, por condados o vecindarios.

Se usa la expresión $\mathrm{H}_{g i}{ }^{\prime}$ para designar el cálculo preliminar del número de UD i en el grupo $g$, y la expresión $\mathrm{H}_{\mathrm{g}}$ para designar la estimación preliminar correspondiente del número total de UD en el grupo g. Se eligen ng conglomerados de cada grupo o estrato $g, g=1,2, \ldots, \mathrm{G}$ con una probabilidad en proporción con el número estimado de UD después del desastre (con o sin reemplazo cuando $n_{g} / N_{g}$ es pequeño). Como se hizo anteriormente, se elabora una lista de las UD en cada uno de los conglomerados seleccionados y se indica el número total mediante la expresión $\mathrm{H}_{g i}$. Poste- riormente se escogen aleatoriamente de la lista (usando un muestreo equiprobabilístico sin reemplazo) $k_{g i}$ UD dentro de cada conglomerado seleccionado en la primera etapa y se entrevista a una persona que viva en esa UD (o a un vecino) para determinar el número de personas que habitan en ella y sus necesidades. Se usa la expresión $C_{g i j}$ para designar el número de personas en la UD $j$ del conglomerado i del grupo $g$, ya que $j=1, \ldots, k_{g i}, i=1, \ldots, n_{g^{\prime}}$ y $g=1, \ldots G$, siendo kgi el número de UD́ que en realidad fueron incluidas en la muestra del conglomerado $i$ del grupo g. Por lo tanto, la población total y su estimación están dadas por la siguiente fórmula:

$$
\mathrm{C}=\sum_{g=1}^{G} \sum_{i=1}^{N_{g}} \sum_{j=1}^{H_{g i}} C_{g i j} \quad \hat{C}=\sum_{g=1}^{G} \sum_{i=1}^{n_{g}} \sum_{j=1}^{k_{g i}} w_{g i} C_{g i j}
$$

donde $w_{g i}=\left(1 / n_{g}\right)\left(H_{g}{ }^{\prime} / H_{g i}{ }^{\prime}\right)\left(H_{g i} / k_{g i}\right)$. La varianza estimada estấ dada por:

$$
\begin{aligned}
\operatorname{Var}(\hat{C}) \cong & \sum_{g=1}^{G}\left(\frac{n_{g}}{n_{g}-1}\right) \\
& \sum_{i=1}^{n_{g}}\left(w_{g i} \sum_{j=1}^{k_{g i}} C_{g i j}-\hat{C}_{g} / n_{g}\right)^{2}
\end{aligned}
$$

donde

$$
\hat{C}_{g}=\sum_{i=1}^{n_{g}} \sum_{j=1}^{k_{g i}} w_{g i} C_{g i j}
$$

es decir, la variabilidad dentro del estrato.

\section{ANEXO 2}

El muestreo sin reemplazo en la etapa 1 cuando $\mathrm{n} / \mathrm{N}$ es grande

Damos por sentado que se ha llevado a cabo una encuesta por conglomerados según el ejemplo en el texto, a base de un muestreo equiprobabilístico sin reemplazo en la etapa 1 y sin estratificación. La estimación puntual de $C$ es la que está dada por la ecuación (3). Si se usan las expresiones indicadas en el texto, la varianza de la población que queda después del desastre se calcula mediante la siguiente fórmula:

$\operatorname{Var}_{2}(\hat{C})$

$$
\begin{aligned}
\cong & \left(1-\frac{n}{N}\right) \frac{n}{n-1} \sum_{i=1}^{n}\left[w_{i} \sum_{j=1}^{k_{i}} C_{i j}-\hat{C} / n\right]^{2} \\
& +\frac{n}{N} \sum_{i=1}^{n}\left(\frac{k_{i}}{k_{i}-1}\right)\left(1-\frac{k_{i}}{H_{i}}\right) \\
& \sum_{j=1}^{k_{i}} w_{i}^{2}\left[C_{i j}-C_{i} / k_{i}\right]^{2}
\end{aligned}
$$

donde

$$
C_{i}=\sum_{j=1}^{k_{j}} C_{i j}
$$

Si $n / N$ es pequeño, es decir, que $=0$, la $\operatorname{Var} 2(\hat{C})$ se reduce a la que está dada por la ecuación (4).
ABSTRACT

A modified cluster-sampling method for post-disaster rapid assessment of needs
The cluster-sampling method can be used to conduct rapid assessment of health and other needs in communities affected by natural disasters. Modelled after WHO's Expanded Programme on Immunization method of estimating immunization coverage, the method has been modified to provide (1) estimates of the population remaining in an area, and (2) estimates of the number of people in the post-disaster area with specific needs. This approach differs from that used previously in other disasters where rapid needs assessments only estimated the proportion of the population with specific needs. We proposed a modified $\mathrm{n} \times \mathrm{k}$ survey design to estimate the remaining population, severity of damage, the proportion and number of people with specific needs, the number of damaged or destroyed and remaining housing units, and the changes in these estimates over a period of time as part of the survey. 
